\title{
The Effect of MicroRNA-101 on Angiogenesis of Human Umbilical Vein Endothelial Cells during Hypoxia and in Mice with Myocardial Infarction
}

\author{
Jun Pang, ${ }^{1,2,3,4}$ Liwen Ye, ${ }^{1,2,5}$ Qingwei Chen $\mathbb{D}^{1},{ }^{1}$ Jian Wang, ${ }^{1}$ Xixi Yang, ${ }^{1}$ Wuyang He, ${ }^{1}$ \\ and Lan Hao ${ }^{2}$ \\ ${ }^{1}$ Department of Geriatrics, The Second Affiliated Hospital of Chongqing Medical University, Chongqing 400010, China \\ ${ }^{2}$ Chongqing Key Laboratory of Ultrasound Molecular Imaging, Ultrasound Department of the Second Affiliated Hospital of \\ Chongqing Medical University, Chongqing 400010, China \\ ${ }^{3}$ Institute of Anorectal Diseases, North Sichuan Medical College, Nanchong 637000, China \\ ${ }^{4}$ Department of Anesthesiology, The Second Clinical College of North Sichuan Medical College, Nanchong Central Hospital, \\ Nanchong 637000, China \\ ${ }^{5}$ State Key Laboratory of Ultrasound Engineering in Medicine Co-Founded by Chongqing and the Ministry of Science \\ and Technology, Chongqing Medical University, Chongqing 400010, China
}

Correspondence should be addressed to Qingwei Chen; chenqwcq@126.com

Received 5 March 2020; Revised 10 June 2020; Accepted 5 August 2020; Published 4 September 2020

Academic Editor: Kimimasa Tobita

Copyright ( 2020 Jun Pang et al. This is an open access article distributed under the Creative Commons Attribution License, which permits unrestricted use, distribution, and reproduction in any medium, provided the original work is properly cited.

\begin{abstract}
Background. Previous studies showed that recanalization and angiogenesis within the infarct region are of vital importance to the survival of myocardial cells during the treatment of acute myocardial infarction (AMI). Methods. In this study, EdU cell proliferation assay, Transwell assay, scratch wound assay, and tube formation assay were used. Twelve bioinformatics analysis packages were used to predict the target genes of miR-101. Target genes were verified by luciferase reporter generation and assay, fluorescent quantitative PCR, and western blotting. Animal model and treatments were detected by M-mode echocardiography and immunofluorescent staining of CD31, Ki67, and $\alpha$-SMA. Results. AgomiR-101 significantly enhanced HUVEC proliferation, migration, and tube formation. A double-luciferase reporter assay revealed that the hsa-miR-101 mimic attenuated the activity of the EIF4E3'-UTR-wt type plasmid by $36 \%$. The expression levels of HIF- $1 \alpha$ and VEGF-A in the scrambled RNA group were significantly lower than those in the EIF4E3 siRNA and agomiR-101 groups. The left ventricular ejection fraction of the AMI+Adv-miR-101 group was significantly higher than that of the AMI+Adv-null and Sham+Adv-null groups. The proliferation of vessel cells in the peripheral infarcted myocardium was higher in the AMI+Adv-miR-101 group than that in the AMI+Adv-null and Sham+Adv-null groups. Conclusion. MiR-101 can promote angiogenesis in the region surrounding the myocardial infarction.
\end{abstract}

\section{Introduction}

Heart failure, caused by acute myocardial infarction (AMI), is a major cause of mortality worldwide. More than three million people suffer from acute ST-segment elevation myocardial infarction every year [1]. In recent years, the incidence of myocardial infarction has increased [2]; thus, it is necessary to explore the pathogenesis of AMI.
Previous studies have shown that recanalization and angiogenesis in the infarct area are vital to the survival of myocardial cells during the treatment of AMI. At present, therapeutic angiogenesis can effectively establish collateral circulation, improve blood supply to the myocardial infarct area, increase the viability of myocardial cells, and improve the condition of myocardial infarction [3]. Some studies [4-7] have shown that multiple angiogenesis stimulating factors are involved in angiogenesis. 
MicroRNAs (miRNAs) are small, highly conserved, endogenous, single-stranded RNA molecules of approximately 19-25 nucleotides that negatively regulate protein expression in various organisms [8]. A number of studies demonstrated that miRNAs have regulatory roles in cardiovascular diseases [9]. In particular, miR-101 functions in the repair and therapeutic regeneration of cardiovascular diseases [10].

The effect of miR-101 on the formation of angiogenesis after AMI has yet to be reported. In the current study, AMI cell and animal models were used to examine whether miR101 plays an important role in the proliferation, migration, and angiogenesis of vascular endothelial cells, as well as the recovery of cardiac function. Moreover, to identify new therapeutic tactics for the treatment of AMI, the targets and signaling pathways of miR-101 in angiogenesis were examined.

\section{Materials and Methods}

2.1. Cell Culture. Human umbilical vein endothelial cells (HUVECs) were cultivated in Dulbecco's modified Eagle medium (DMEM), containing 1\% fetal bovine serum (FBS) and penicillin/streptomycin, and were maintained at $37^{\circ} \mathrm{C}$ in a $5 \% \mathrm{CO}_{2}$ incubator.

2.2. EdU Cell Proliferation Assay. When the cell fusion reached $80 \%$, the cells were divided into three groups and agomiR-101, antagomiR-101, and scrambled RNA were added to the culture medium at a final concentration of $200 \mathrm{nM}$. The culture medium also contained $100 \mu \mathrm{M} \mathrm{CoCl}_{2}$ and $10 \%$ FBS. HUVEC proliferation was detected $48 \mathrm{~h}$ after cultivation using an EdU assay. A fluorescent microscope was used to observe the samples and obtain images.

2.3. Scratch Wound Assay. Approximately $3 \times 10^{5}$ HUVECs were inoculated on a 6-well plate. After the HUVECs reached $70 \%$ confluence, they were transfected with agomiR-101, antagomiR-101, or scrambled RNA in $100 \mu \mathrm{M} \mathrm{CoCl}_{2}$. At $48 \mathrm{~h}$ post-transfection, three parallel lines were drawn on the back of the 6-well plate using a marker pen and the cell layer was scratched perpendicular to the transverse lines using a $100 \mu \mathrm{l}$ Finnpipette. The cells were then washed three times with $0.01 \mathrm{M}$ phosphate-buffered saline (PBS) and cultured in DMEM containing $1 \%$ fetal calf serum at $37^{\circ} \mathrm{C}$ and $5 \% \mathrm{CO}_{2}$. The intersection points between the scratches and the marked lines were photographed after $24 \mathrm{~h}$ and scratch wound healing was determined.

2.4. Transwell Assay. After starvation for $24 \mathrm{~h}$, the cell suspension was collected, centrifuged, and washed twice. A $400 \mu \mathrm{l}$ aliquot of cell suspension was then added to the upper chamber of a Transwell plate. NIH3T3 culture solution $(600 \mu \mathrm{l})$ was added to the lower chamber as the chemotaxis liquid. The culture plate was incubated for $48 \mathrm{~h}$ at $37^{\circ} \mathrm{C}$ in a $5 \% \mathrm{CO}_{2}$ incubator. The cells on the filter membrane of the Transwell insert were carefully removed and subsequently fixed with methanol for $5 \mathrm{~min}$ and rinsed three times with PBS for 3 min per rinse. Hematoxylin and eosin staining was performed, and the numbers of penetrating cells were determined in five randomly selected high power fields per filter membrane.

2.5. Tube Formation Assay. Precooled Matrigel (300 $\mu \mathrm{l} /$ well) was added to a 24 -well plate and incubated at $37^{\circ} \mathrm{C}$ for $30 \mathrm{~min}$ to solidify the Matrigel. HUVECs were transfected with agomiR-101, antagomiR-101, or scrambled RNA at a final concentration of $200 \mathrm{nM}$ for $48 \mathrm{~h}$ in $100 \mu \mathrm{M} \mathrm{CoCl}$ and then inoculated on the surface of the solidified Matrigel. After mixing, the sample was incubated for $6 \mathrm{~h}$ at $37^{\circ} \mathrm{C}$ and $5 \% \mathrm{CO}_{2}$. Tube formation was observed, and the circumferences of the tubes were measured in five high power fields.

2.6. Bioinformatics Analyses. Twelve bioinformatics analysis packages were used to predict the target genes of miR-101, namely, miRanda, miRSystem, miRDB, RNAhybrid, miRMap, TargetScan, miRNAsMap, miRWalk, PicTar, PITA, DIANA-microT, and RNA22. Based on the sequence of mature miRNA-101 (UACAGUACUGUGAUAACUGAA), the gene encoding eukaryotic translation initiation factor E3 (EIF4E3) was predicted as a target.

\subsection{Luciferase Reporter Generation and Assay}

2.7.1. Construction of the Luciferase Reporter Plasmids. Luciferase reporter plasmids harboring the wild-type $3^{\prime}$ -UTR of EIF4E3, containing the predicted miR-101-binding site (EIF4E3' -UTR-wt; GUACUGU), or a mutated version of the UTR containing a point mutation in the miR-101 recognition site (EIF4E3'-UTR-muta; GUACUGU), were constructed.

2.7.2. Plasmid Transfection. Human 293 T cells were inoculated on a 48 -well plate and incubated for $24 \mathrm{~h}$ at $37^{\circ} \mathrm{C}$ and $5 \% \mathrm{CO}_{2}$ until the cell density reached $80 \%$. Lipofectamine $3000(1 \mu \mathrm{l})$ was added to $25 \mu \mathrm{l}$ of Opti-MEM, and the mixture was stored at room temperature for $5 \mathrm{~min}$. Plasmid $(0.2 \mu \mathrm{g})$ was added to another $25 \mu \mathrm{l}$ aliquot of Opti-MEM, and the mixture was stored at $25^{\circ} \mathrm{C}$ for $5 \mathrm{~min}$. Additionally, agomiR-101 or scrambled RNA $(7.5 \mathrm{pmol})$ was added to $25 \mu \mathrm{l}$ of Opti-MEM, and the mixture was incubated at room temperature for $5 \mathrm{~min}$. The transfection reagent, plasmid, and agomiR-101 or scrambled RNA solutions were mixed and incubated at room temperature for $20 \mathrm{~min}$. Culture medium $(50 \mu \mathrm{l} /$ well $)$ and the transfection complex ( $50 \mu \mathrm{l} /$ well) were added to each well of the 48 -well plate (three wells per group), and the cells were incubated at $37^{\circ} \mathrm{C}$ and $5 \% \mathrm{CO}_{2}$. The transfection mixture was subsequently replaced with $200 \mu \mathrm{l}$ of fresh medium and the cells were incubated at $37^{\circ} \mathrm{C}$ and $5 \% \mathrm{CO}_{2}$ for a further $48 \mathrm{~h}$.

2.7.3. Luciferase Assay. A $200 \mu$ l aliquot of cell lysis buffer was added to each well of a 48 -well plate and incubated at room temperature for $8 \mathrm{~min}$. The sample was then centrifuged at $4^{\circ} \mathrm{C}$ and $10000 \mathrm{~g}$ for $5 \mathrm{~min}$, and the lysis supernatant was collected. A $20 \mu \mathrm{l}$ aliquot of the lysis supernatant and a $100 \mu \mathrm{l}$ aliquot of firefly luciferase working liquid were added to each well of a 96-well plate, and the luciferase signal was measured. As a control, a $100 \mu \mathrm{l}$ aliquot of Renilla luciferase working fluid was added to each well. 
2.8. Fluorescent Quantitative PCR. Total RNA was extracted from HUVECs using TRIzol reagent (Takara, China) and reverse transcribed using the miRNA reversible transcription PCR kit (Promega, USA). The fluorescent quantitative PCR assay was performed using the following primers: EIF4E3, Forward: TGCAAAGGGTGGCGTATGGAAG, Reverse: TCTTCTCGGTCCCGAACACTGA; EIF4E1, Forward: AT GCCTGGCTGTGACTACTCAC, Reverse: GAGGTCACT TCGTCTCTGCTGT; VEGF-A, Forward: TTGCCTTGCTG CTCTACCTCCA, Reverse: GATGGCAGTAGCTGCGCT GATA; HIF- $1 \alpha$, Forward: TTGCCTTGCTGCTCTACCT CCA, Reverse: GATGGCAGTAGCTGCGCTGATA; and GAPDH, Forward: GTCTCCTCTGACTTCAACAGCG, Reverse: ACCACCCTGTTGCTGTAGCCAA.

A fluorescent qRT-PCR detection system (Bio-Rad, USA) was used for amplification, and a fluorescent quantitation PCR kit (Promega, USA) was used for detection of the target genes. The reactions were performed using SYBR green mix, cDNA, each primer, and RNase-free water. The cycling conditions were as follows: denaturation at $95^{\circ} \mathrm{C}$ for $10 \mathrm{~min}$, followed by 40 cycles at $95^{\circ} \mathrm{C}$ for $2 \mathrm{~s}$ and $60^{\circ} \mathrm{C}$ for $20 \mathrm{~s}$, and then a final extension at $70^{\circ} \mathrm{C}$ for $10 \mathrm{~s}$. The expression of $\beta$-actin was used as an internal reference, and the relative expression of miR-101 was calculated using the $2^{-\Delta \Delta} \mathrm{Ct}$ method.

2.9. Western Blotting. Protein was extracted from HUVECs using RIPA Lysis Buffer (Millipore, USA), and protein concentration was measured using the BCA Protein Quantitative Kit (Beyotime, China). The proteins were separated by SDS-PAGE and then transferred to a PVDF membrane. The following primary antibodies were used: rabbit monoclonal anti-HIF-1 $\alpha$ (ab179483; Abcam, UK), rabbit polyclonal anti-VEGF-A (ab46154; Abcam), rabbit polyclonal antiEIF4E3 (17282-1-AP; Proteintech, USA), rabbit polyclonal anti-EIF4E1 (17282-1-AP; Cell Signaling Technology, USA), and rabbit polyclonal anti-GAPDH (10494-1-AP; Proteintech). After incubation with the primary antibodies, the membrane was incubated with horseradish peroxidase-labeled goat-anti-rabbit IgG (ZB-2301, ZSGB-Bio, China). The immunoproteins were detected using the Hypersensitivity ECL Luminescence Kit (Beyotime), and band densities were quantified using Quantity One v4.6.2 (Bio-Rad).

2.10. Animal Model and Treatments. Animals were used in strict accordance with the Guide for the Care and Use of Laboratory Animals under the approval of the Institutional Ethics Committee of Chongqing Medical University (Permit No. SCXK (Chongqing) 2007-0001) and the State Science and Technology Commission of China. The present study made use of 22 eight-week-old C57BL/6 mice, bred in the Animal Center of Chongqing Medical University. Mice were fed with a standard chow diet. The AMI model was established by ligating the anterior descending branch of the coronary artery via thoracotomy. The mice were randomly divided into the AMI+Adv-miR-101 $(n=10$, death $=3)$, AMI+Adv-null $(n=11$, death $=4)$, and Sham+Adv-null $(n=10$, death $=2)$ groups. A viral vector containing miR101 , or a null viral vector (negative control), was injected into

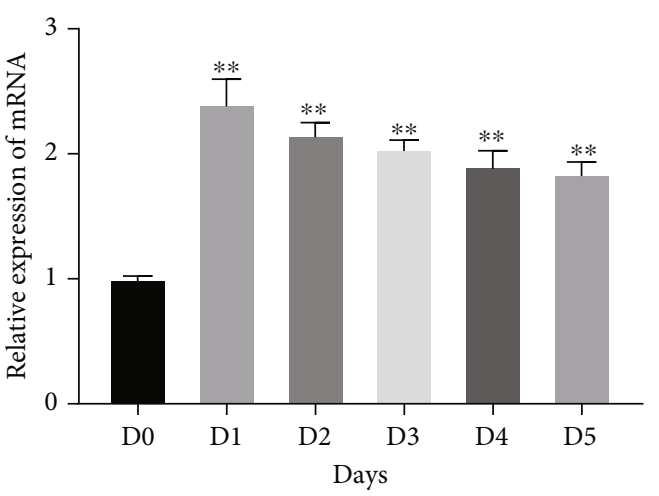

FIgure 1: The miR-101 expressions in HUVECs with $\mathrm{CoCl}_{2}$ detected by qRT-PCR for 5 days $\left({ }^{* *} P<0.01\right.$ vs. D0).

the left ventricular anterior wall infarction and peripheral infarction area at three points. After two weeks, the mice were euthanized by cervical dislocation. The hearts were removed and washed with saline. Paraffin and frozen sections were then prepared, and the latter were stored at $-20^{\circ} \mathrm{C}$.

2.11. Parasternal Short-Axis M-Mode Echocardiography. After 14 days of AMI, M-mode echocardiography was used to detect changes in cardiac function in the C57BL/6 mice. A Vivid E9 diasonograph (GE Healthcare, USA) was used for detection. The transducer frequency was $15 \mathrm{MHz}$, and the depth was $1.0-1.5 \mathrm{~cm}$. Mice were intraperitoneally injected with $1 \%$ pentobarbital sodium at a dose of $50 \mathrm{mg} / \mathrm{kg}$ and then fixed on the operating plate in a supine position. The fur was removed from the chest, and the coupling agent was evenly coated. The ultrasonic probe was then applied carefully to the chest, and images were collected.

2.12. Immunofluorescent Staining of CD31, Ki67, and $\alpha$-SMA. For immunostaining, frozen sections of the mouse hearts were removed from the $-20^{\circ} \mathrm{C}$ freezer, thawed at room temperature for $30 \mathrm{~min}$, and washed three times with $0.01 \mathrm{M}$ PBS (5 min per wash). A $2 \%$ BSA solution or immunostaining sealant was dripped onto the tissue sections; thereafter, the sections were sealed at $37^{\circ} \mathrm{C}$ for $1 \mathrm{~h}$ and dried with filter paper. Subsequently, the anti-CD31, anti- $\alpha$-SMA, and antiKi67 primary antibodies were diluted at a ratio of $1: 100$ with $0.1 \%$ Triton X-100 and dripped onto the tissue sections. The sections were incubated overnight at $4^{\circ} \mathrm{C}$ and then washed three times with $0.01 \mathrm{M}$ PBS ( $20 \mathrm{~min}$ per wash). Next, the secondary antibodies were dripped into each tissue section, and the sections were bathed in water at $37^{\circ} \mathrm{C}$ in the dark. The samples were then washed three times with $0.01 \mathrm{M}$ PBS (10 min per wash). The sections were stained with DAPI for $10 \mathrm{~min}$ at room temperature in the dark. An antifluorescence quenching agent was then dripped onto the samples, and cover glasses were applied. Images were obtained using a fluorescence microscope.

2.13. Statistical Analysis. Image-Pro Plus software was used to analyze the proliferation rate, apoptosis rate, and vessel density of endothelial cells. Statistical analyses were performed using GraphPad Prism 6.0 software. All data are 


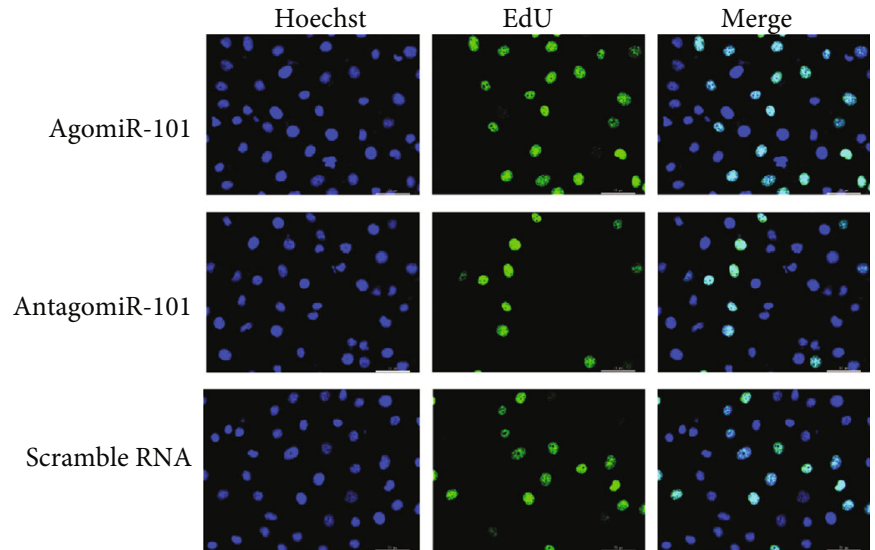

(a)

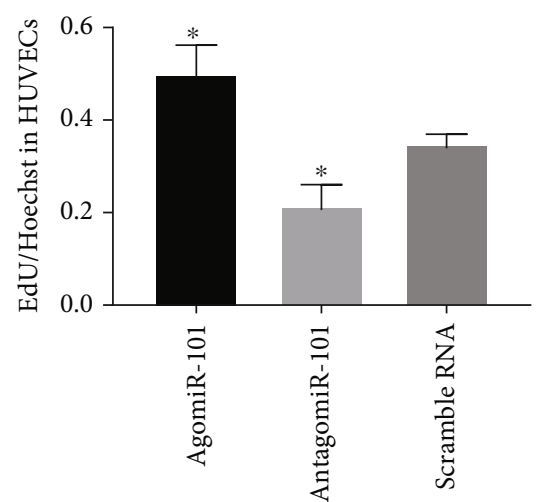

(b)

FIgURE 2: (a) Photomicrographs show the EdU cell proliferation assay of HUVECs transfected with agomiR-101, antagomiR-101, or negative control (scrambled RNA). (b) Bar graphs show the proliferation rate of HUVECs detected by EdU cell proliferation assay $\left({ }^{*} P<0.05\right.$ vs. scrambled RNA).

presented as the mean \pm standard deviation. A one-way analysis of variance (ANOVA) was used when analyzing single variables, a multiway ANOVA was used to analyze two or more variables, and between-group comparisons were performed using the Kruskal-Wallis tests. $P<0.05$ was considered statistically significant.

\section{Results}

3.1. The Effect of Hypoxia on miR-101 Expression in HUVECs. Quantitative RT-PCR analyses were used to examine the expression levels of miR-101 in HUVECs exposed to hypoxia for $0,1,2,3,4$, or 5 days. miR-101 expression increased rapidly from the first day of exposure. On days $2-5$, miR101 expression was significantly higher than at the baseline (Figure 1).

3.2. The Effect of AgomiR-101 on the Proliferation, Migration, and Tube Formation of HUVECs. We examined the effects of agomiR-101, antagomiR-101, and a scrambled RNA (negative control) on the proliferation, migration, and tube formation of HUVECs. An EdU assay was used to determine cell proliferation. The number of EdU-labeled HUVECs in the agomiR-101 group was significantly higher than that in the scrambled RNA group. In addition, the number of EdUlabeled cells in the scrambled RNA group was significantly higher than that in the antagomiR-101 group (Figures 2(a) and $2(\mathrm{~b}))$.

In the scratch wound assay, agomiR-101 and antagomiR101 promoted and inhibited HUVEC migration during wound healing, respectively (Figures 3(a) and 3(d)). In addition, a Transwell assay showed that the number of migrating cells in the agomiR-101 group was significantly higher than that in the scrambled RNA group, and the number of migrating cells in the scrambled RNA group was significantly higher than that in the antagomiR-101 group (Figures 3(b) and $3(\mathrm{e}))$. A tube formation assay revealed that the blood vessel in the agomiR-101 group was significantly longer than in the antagomiR-101 group, and the blood vessel in the
antagomiR-101 group was significantly shorter than in the scrambled RNA group (Figures 3(c) and 3(f)). Overall, the results of these experiments show that agomiR-101 significantly enhanced HUVEC proliferation, migration, and tube formation.

3.3. The Effect of miR-101 on EIF4E3 Expression. Various miRNA target prediction packages identified EIF4E3 as a potential target of miR-101; thus, we examined the effects of miR-101 on EIF4E3 expression in HUVECs. Two luciferase reporter plasmids containing the wild-type $3^{\prime}$-UTR of EIF4E3 (EIF4E3'-UTR-wt) or a mutated version of the UTR containing a point mutation in the $\mathrm{miR}-101$ recognition site (EIF4E3' -UTR-muta) were generated and transfected into HUVECs. The cells were also transfected with a negative control or hsa-miR-101 mimic. The results of a doubleluciferase (firefly and Renilla) reporter assay revealed that the hsa-miR-101 mimic attenuated the activity of the EIF4E3' -UTR-wt type plasmid by $36 \%$, but had no effect on the activity of the EIF4E3' -UTR-muta plasmid (Figure 4).

3.4. The Effects of an EIF4E3 siRNA and AgomiR-101 on the Expression Levels of EIF4E3, EIF4E1, HIF-1 $\alpha$, and VEGF-A. HUVECs were transfected with scrambled RNA, scrambled RNA+EIF4E3 siRNA, agomiR-101, and agomiR-101+ EIF4E3 siRNA for $48 \mathrm{~h}$. Subsequently, western blotting and qRT-PCR were used to detect the expression levels of EIF4E3, EIF4E1, hypoxia-inducible factor $1 \alpha$ (HIF-1 $\alpha$ ), and vascular endothelial growth factor A (VEGF-A) (Figures 5(a)-5(c)). The expression levels of EIF4E3 in the scrambled RNA+EIF4E3 siRNA and agomiR-101 groups were significantly lower than those in the scrambled RNA group, but they were higher than those in the agomiR-101 +EIF4E3 siRNA group. However, EIF4E1 expression was not affected by agomiR-101 or the downregulation of EIF4E3. The expression levels of HIF- $1 \alpha$ and VEGF-A in the scrambled RNA group were significantly lower than those in the EIF4E3 siRNA and agomiR-101 groups. There were no significant differences in HIF- $1 \alpha$ and VEGF-A 

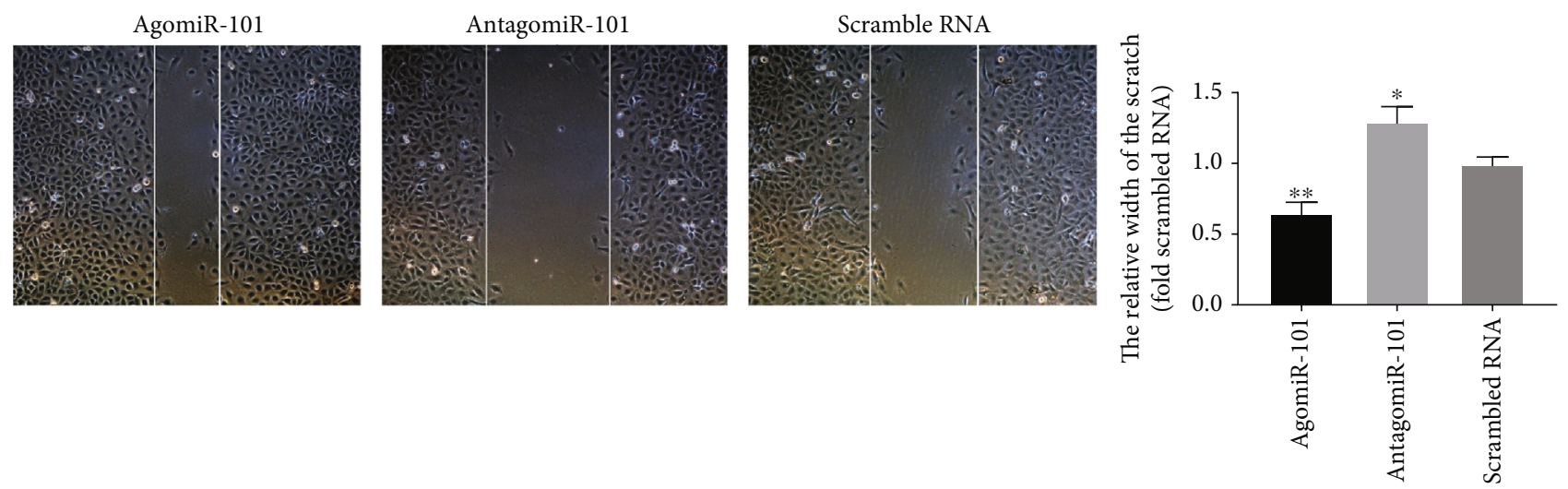

(a)
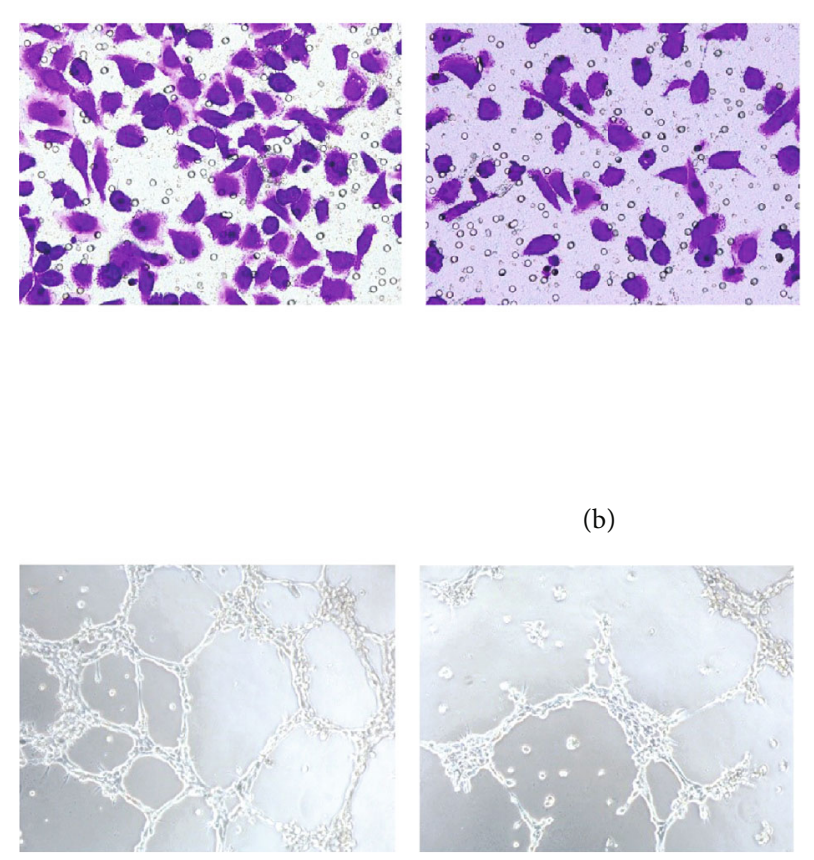

(c)
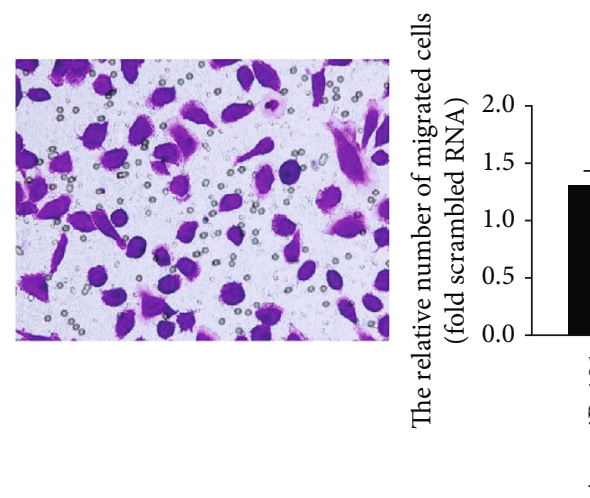

(d) (b)
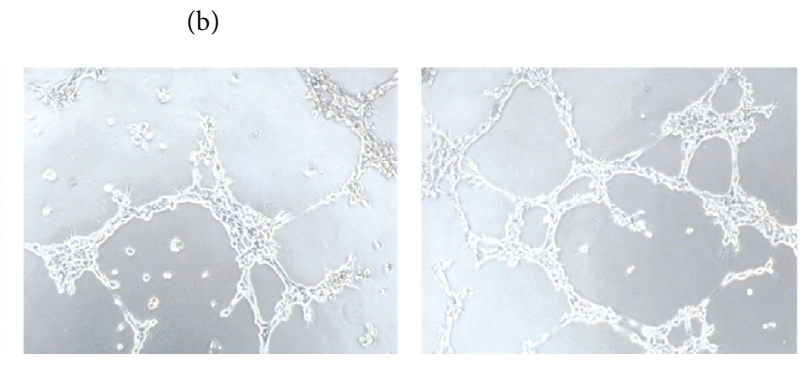

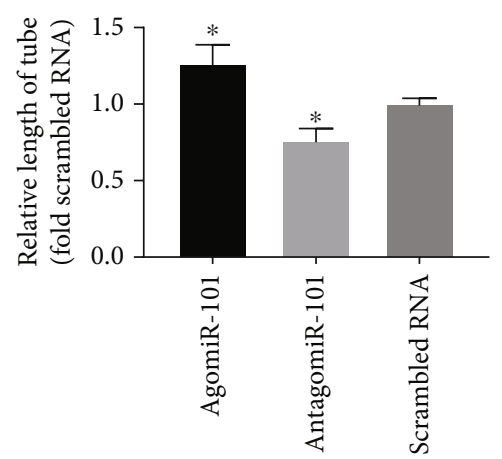

(f)

Figure 3: Photomicrographs ( $a, b$, and c) show the scratch wound assay, Transwell assay, and tube formation assay of HUVECs transfected with agomiR-101, antagomiR-101, or negative control (scrambled RNA). Bar graphs (d, e, and f) show the results of scratch wound assay, Transwell assay, and tube formation assay ( ${ }^{*} P<0.05$ vs. scrambled RNA; ${ }^{* *} P<0.01$ vs. scrambled RNA).

expression between the EIF4E3 siRNA group and the agomiR-101 groups; however, the expression level of HIF$1 \alpha$ and VEGF-A in the agomiR-101+EIF4E3 siRNA group was higher than that in the agomiR-101 group, as well as the EIF4E3 siRNA group.

3.5. The Effects of an AgomiR-101, Antagomir-101, and Scrambled RNA on the Expression Levels of EIF4E3, EIF4E1, $H I F-1 \alpha$, and VEGF-A. HUVECs were transfected with agomiR-101, antagomiR-101, and scrambled RNA for $48 \mathrm{~h}$. Western blotting and qRT-PCR were used to detect the expression levels of EIF4E3, EIF4E1, hypoxia-inducible factor $1 \alpha$ (HIF-1 $\alpha$ ), and vascular endothelial growth factor $\mathrm{A}$ (VEGF-A) (Figures 6(a)-6(c)). The expression levels of EIF4E3 in the agomiR-101 groups were significantly lower than those in the scrambled RNA group, but were higher than those in the antagomiR-101 group. However, EIF4E1 expression was not affected by agomiR-101 or antagomir101. The expression levels of HIF- $1 \alpha$ and VEGF-A in the scrambled RNA group were significantly lower than those in the agomiR-101 group, but were higher than those in the antagomiR-101 group. 


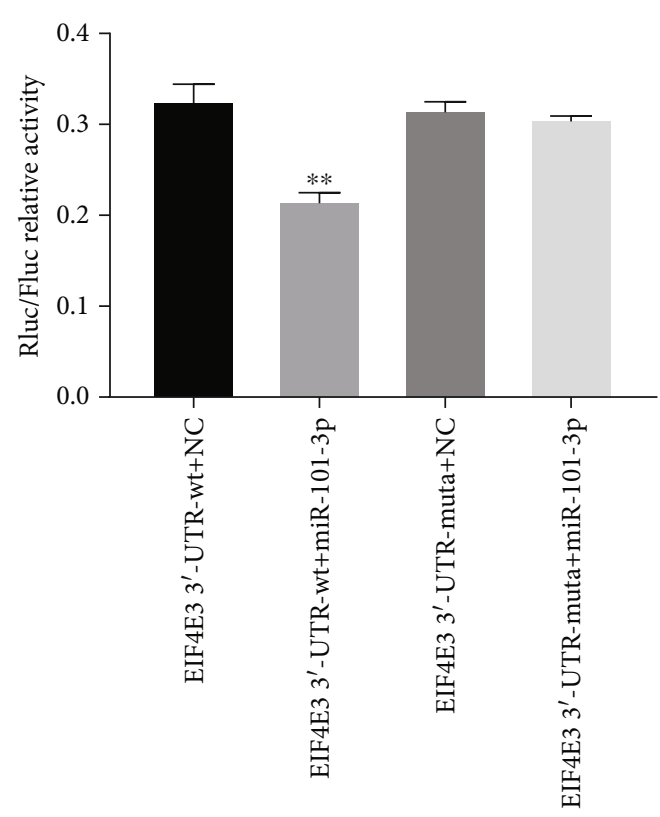

Figure 4: Double-luciferase reporter gene was used to detect the inhibition level of miR-101 targeting on EIF4E3 $\left({ }^{* *} P<0.01\right.$ vs. EIF4E3' -UTR-wt+NC).

3.6. The Effect of miR-101 on Cardiac Function in C57BL/6 Mice. Two weeks after the establishment of AMI or sham treatment as a control, M-mode echocardiography was used to detect the cardiac function of $\mathrm{C} 57 \mathrm{BL} / 6$ mice that were injected at the left ventricular anterior wall infarction and peripheral infarction area, with a viral vector containing miR-101 (Adv-miR-101) gene or a null viral vector as a negative control (Adv-null). The left ventricular ejection fraction (LVEF) of the AMI+Adv-miR-101 group was significantly higher than that of the AMI+Adv-null and Sham+Adv-null groups, and the LVEF of the Sham+Adv-null group was significantly higher than that of the AMI+Adv-null group (Table 1). Similarly, the left ventricular shortening fraction of mice in the AMI+Adv-miR-101 group was significantly higher than that of mice in the AMI+Adv-null and Sham+Adv-null groups, and that of mice in the Sham+ Adv-null group was significantly higher than that in the AMI+Adv-null group. Finally, the left ventricular endsystolic diameter of mice in the $\mathrm{AMI}+\mathrm{Adv}-\mathrm{miR}-101$ group was significantly lower than that in the AMI+Adv-null and Sham+Adv-null groups. Overall, the results indicated that miR-101 improved the cardiac function of AMI mice.

3.7. The Effect of miR-101 on the Proliferation of Vascular Endothelial Cells in Myocardial Infarction Mice. Immunofluorescent staining was used to evaluate the proliferation of vascular endothelial cells in AMI and sham-treated C57BL/6 mice after injection of Adv-miR-101 or Adv-null for two weeks (Figures 7(a) and 7(b)). The proliferation of these cells in the peripheral infarcted myocardium was significantly higher in the AMI+Adv-miR-101 group than that in the AMI+Adv-null and Sham+Adv-null groups.
3.8. The Effect of miR-101 on Myocardial Vessel Density in Myocardial Infarction Mice. CD31 immunofluorescent staining was used to examine the myocardial vessel densities in the AMI+Adv-miR-101, AMI+Adv-null, and Sham+Advnull groups (Figures 8(a) and 8(c)). The vessel density of the peripheral infarcted myocardium in the AMI+AdvmiR-101 group was significantly higher than that in the $\mathrm{AMI}+\mathrm{Adv}$-null and Sham+Adv-null groups. In addition, the vessel density of the peripheral infarcted myocardium in the AMI+Adv-null group was higher than that in the Sham+Adv-null group. Immunofluorescent staining of $\alpha$ SMA was also used to assess the density of arterioles (Figures $8(\mathrm{~b})$ and $8(\mathrm{~d})$ ). The arteriole density of the peripheral infarcted myocardium was higher in the AMI+AdvmiR-101 group than that in the AMI+Adv-null and Sham+ Adv-null groups. There were no significant differences between the arteriole densities of the peripheral infarcted myocardium in the Sham+Adv-null group and AMI+Advnull groups.

\section{Discussion}

AMI is a clinical syndrome characterized by rupture of atheromatous plaque and acute arterial occlusion, resulting in myocardial necrosis. Recirculation of the residual blood vessels in the infarcted area and rapid angiogenesis are of vital importance to cardiac repair after myocardial infarction. The establishment of artificial collateral circulation significantly improves the survival rate of AMI patients, by enhancing both the blood supply to the infarcted area and the viability of myocardial cells $[11,12]$. Recent studies demonstrated that promoting angiogenesis in ischemic areas is effective for the treatment of ischemic diseases. Therapeutic angiogenesis, which involves inducing local reangiogenesis in ischemic areas and forms a compensatory collateral circulation, promoting the opening of new blood vessels and self-healing of related cells, can effectively alleviate myocardial ischemia.

A large number of studies demonstrated that miRNAs play regulatory roles in cardiovascular diseases. For example, antagomiRs or recombinant adenoviruses targeting miR-24 improve the recovery of cardiac function in myocardial infarction mice, by modulating the pathway involving endothelial nitric oxide synthase, the endothelial cell transcription factor GATA-2, and the serine/threonine protein kinase PAK4 [13]. In addition, Kim et al. [10] found that the expression of miR-101 in vascular endothelial cells is markedly increased under hypoxic conditions. miRNA-101 promotes endothelial cell growth via the Cul3/Nrf2/heme oxygenase (HO-1)/VEGF pathway, indicating that it plays an important role in the regulation of angiogenesis. During cell and tissue adaptation to hypoxia, genes involved in angiogenesis, cell proliferation, and glucose metabolism are activated. In eukaryotic cells, HIF-1 is the main transcription factor activated by hypoxia and the major regulator of oxygen homeostasis [14].

In the current study, bioinformatics analyses predicted EIF4E3 to be the target of miR-101. EIF4E3 is a eukaryotic translation initiation factor that competitively inhibits the 


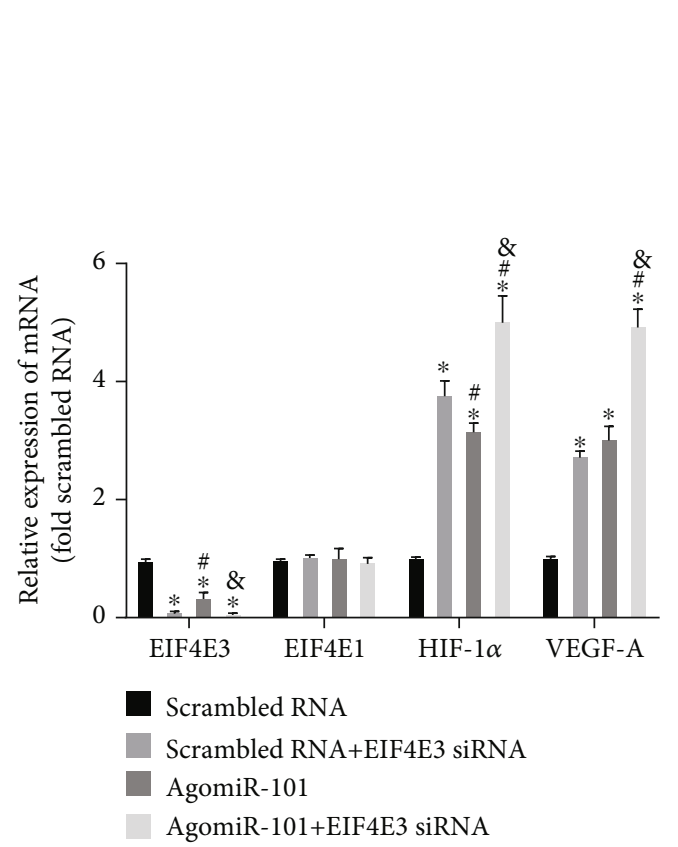

(a)

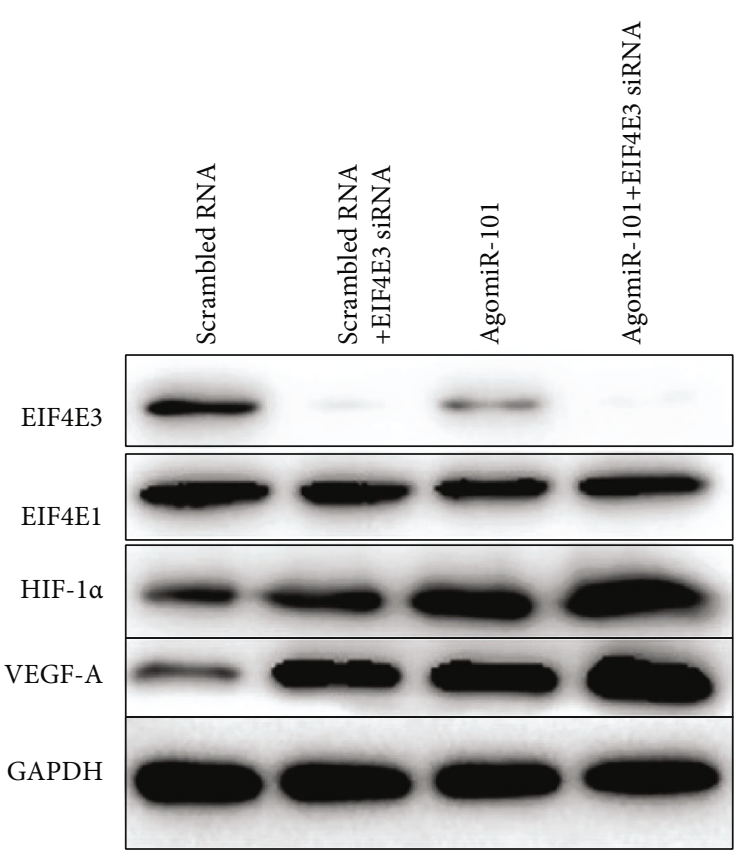

(b)

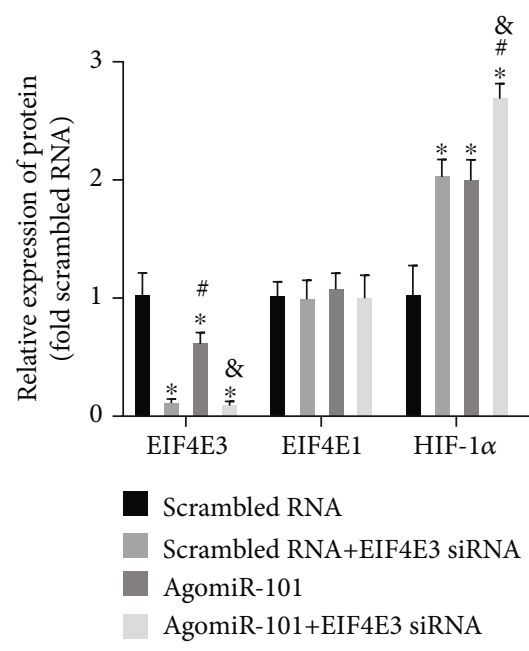

(c)

FIGURE 5: Bar graphs show the effect of scrambled RNA, scrambled RNA+EIF4E3 siRNA, agomiR-101, and agomiR-101+EIF4E3 siRNA on the mRNA (a) and protein expression (b, c) of EIF4E3, EIF4E1, HIF- $1 \alpha$, and VEGF-A $\left({ }^{*} P<0.01\right.$ vs. scrambled RNA; ${ }^{*} P<0.01$ vs. scrambled RNA+EIF4E3 siRNA; ${ }^{\&} P<0.01$ vs. agomiR-101).

expression of EIF4E1, an important vascular stimulating factor in angiogenesis. By attenuating the activation of the HIF-1 signaling pathway, EIF4E3 inhibits the formation and development of newly born capillaries, subsequently obstructing angiogenesis $[15,16]$. EIF4E3 is downregulated in rapidly proliferating tumor cells [17], whereas EIF4E1 (formerly known as EIF4E) is abundantly expressed in tumor cells $[18,19]$, although it is also present in most cells and plays an important role in angiogenesis. Multiple studies have shown that inhibiting EIF4E can inhibit cell proliferation and angiogenesis $[20,21]$. Osborne et al. found that EIF4E3 and EIF4E1 could be integrated into the m7G-cap structure of mRNAs, and that these factors exhibited a competitive relationship. Therefore, EIF4E3 can inhibit the abil- ity of EIF4E1 to promote cell survival and proliferation. In addition, EIF4E3 can also affect mRNA transfer out of the nucleus, thereby inhibiting cell proliferation $[15,16]$. Landon et al. found that MNK1/2 inhibits the phosphorylation of EIF4E1 and promotes the expression of EIF4E3, and proposed that MNK1/2 controls cell proliferation by modulating the levels of these translation initiation factors [22]. EIF4E may bind to the cap structure of the HIF-1 $\alpha$ mRNA and promote the translation of HIF- $1 \alpha$ mRNA into HIF- $1 \alpha$ [23-25]. Apart from EIF4E3, the cytokines regulating EIF4E1 were regulated by EIF4E-BPs [26]. Therefore, the ability of EIF4E1 to promote the translation of RNA could be improved; however, this kind of ability was still limited and would not be enlarged indefinitely. 


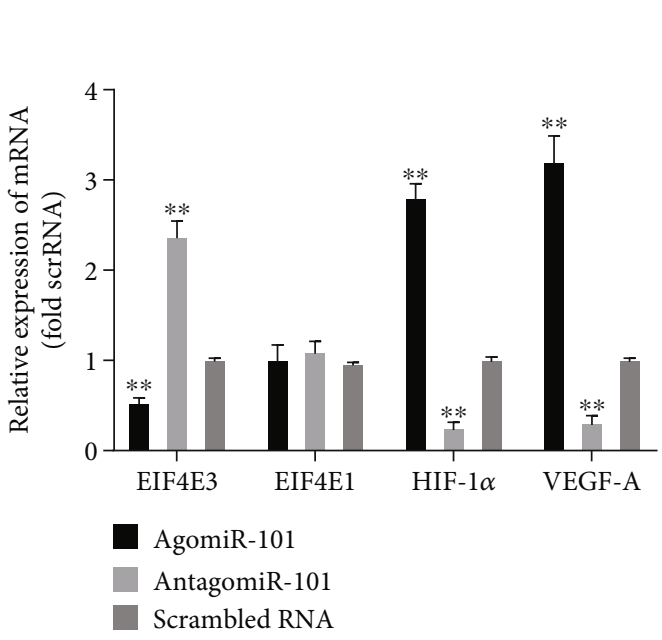

(a)

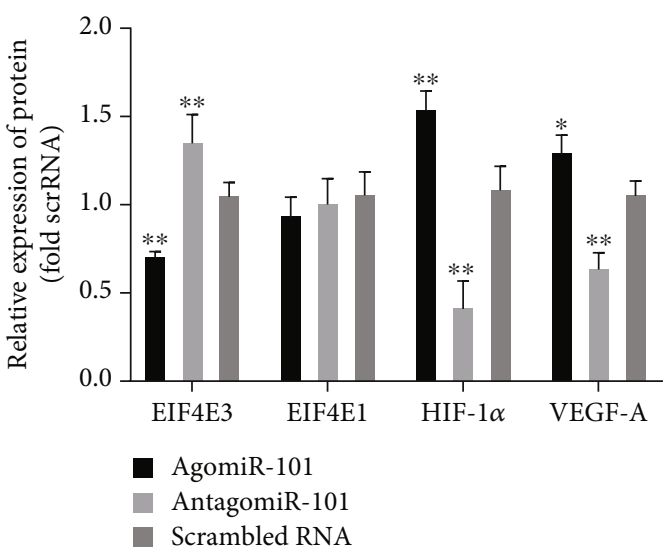

(c)

FIGURE 6: Bar graphs show the effect of agomiR-101, antagomiR-101, and scrambled RNA on the mRNA (a) and protein expression (b, c) of EIF4E3, EIF4E1, HIF- $1 \alpha$, and VEGF-A $\left({ }^{*} P<0.05\right.$ vs. scrambled RNA; ${ }^{* *} P<0.01$ vs. scrambled RNA).

TABLE 1: Various parameters of cardiac function in mice by $M$ type ultrasound.

\begin{tabular}{lccc}
\hline & AMI+Adv-miR-101 & AMI+Adv-null & Sham+Adv-null \\
\hline Interventricular septal diameter $(\mathrm{mm})$ & $0.80 \pm 0.08$ & $0.84 \pm 0.07$ & $0.83 \pm 0.06$ \\
Left ventricular end-diastolic diameter $(\mathrm{mm})$ & $2.69 \pm 0.48$ & $2.87 \pm 0.33^{* *}$ & $2.25 \pm 0.12$ \\
Left ventricular posterior wall diameter $(\mathrm{mm})$ & $0.98 \pm 0.29$ & $0.98 \pm 0.16$ & $0.79 \pm 0.21$ \\
Left ventricular end-systolic diameter $(\mathrm{mm})$ & $1.45 \pm 0.27^{* *}, \#$ & $1.9 \pm 0.22^{* *}$ & $0.87 \pm 0.07$ \\
Left ventricular end-diastolic volume (ml) & $0.06 \pm 0.02^{*}$ & $0.06 \pm 0.02^{* *}$ & $0.03 \pm 0.01$ \\
LV ejection fraction (\%) & $82.86 \pm 4.77^{* *}, \# \#$ & $69.11 \pm 9.81^{* *}$ & $93.89 \pm 1.08$ \\
Fractional shortening (\%) & $45.69 \pm 5.11^{* *}, \# \#$ & $33.72 \pm 6.41^{* *}$ & $61.43 \pm 2.17$ \\
Stroke volume (ml) & $0.05 \pm 0.02$ & $0.05 \pm 0.02$ & $0.03 \pm 0.01$ \\
\hline
\end{tabular}

${ }^{*} P<0.05$ vs. Sham + Adv-null. ${ }^{* *} P<0.01$ vs. Sham+Adv-null. ${ }^{\#} P<0.05$ vs. AMI+Adv-null. ${ }^{\# \#} P<0.01$ vs. AMI+Adv-null.

During the adaptation of cells and tissues to hypoxia, the genes involved in angiogenesis, cell proliferation, and glucose metabolism are activated. In eukaryotic cells, HIF-1 is the main transcription factor activated by hypoxia and the major regulator of oxygen homeostasis [14]. HIF-1 was originally discovered as a transcription factor that regulates the expression of erythropoietin in the blood under hypoxic conditions
$[27,28]$. There are two different subunits of HIF-1, namely, $\alpha$ and $\beta[29,30]$. To date, three HIF- $\alpha$ isomers have been identified: HIF- $1 \alpha$, HIF- $2 \alpha$ (also known as endothelial PAS protein, and HIF- $3 \alpha$ inhibiting PAS protein), and HIF- $3 \alpha$. The second subtype, HIF- $2 \alpha$, is expressed in the endothelial cells, lung, and cartilage, and shares $48 \%$ amino acid sequence homology with HIF- $1 \alpha$. The third subtype, HIF-3 $\alpha$, (also 


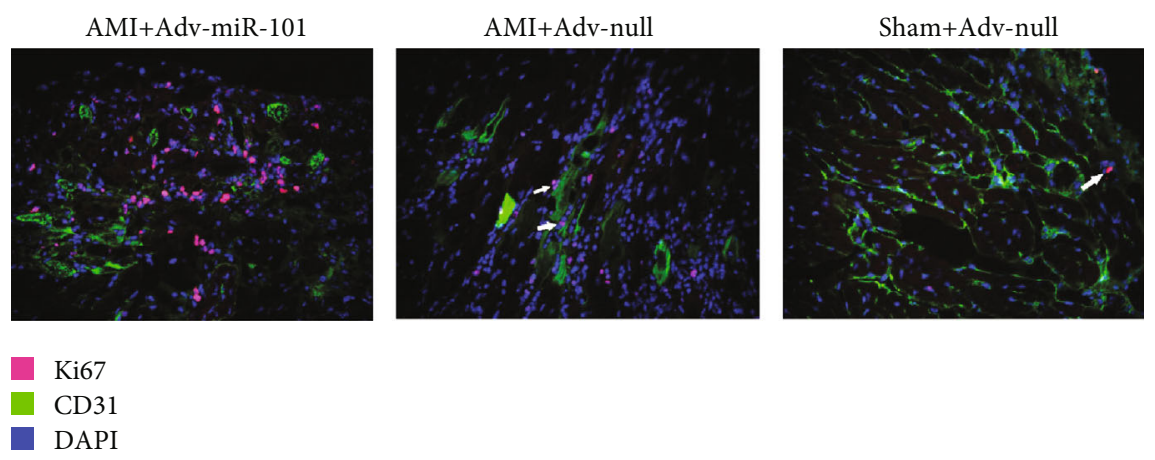

(a)

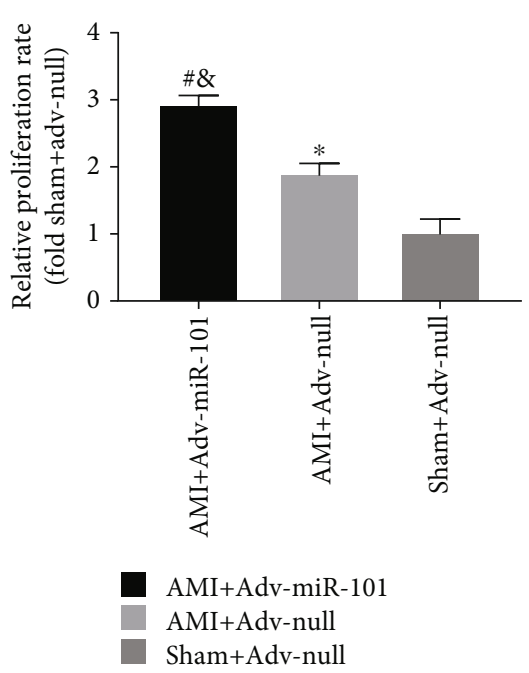

(b)

Figure 7: Photomicrographs (a) and bar graphs (b) show the proliferation of vascular endothelial cells of the peripheral infarcted

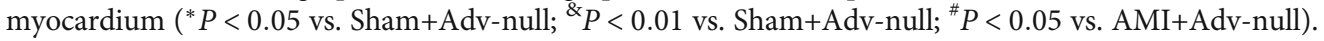

known as PAS inhibition protein), inhibits the binding of HIF-1 $\alpha$ to DNA [31-33]. HIF-1 participates in multiple angiogenic processes. During capillary formation under hypoxic conditions, HIF-1 can directly or indirectly regulate angiogenic factors and cytokines. Enhancing the activity of HIF-1 is imperative to the treatment of ischemic diseases. As mentioned above, angiogenesis is a multistep process [34]; first, hypoxia and HIF-1 directly stimulate the expression of VEGF and its receptor, and then a new angiogenesis process is initiated. Second, the extracellular matrix is degraded by metalloproteinases, allowing migrating endothelial cells to form tubes. Further, HIF- $1 \alpha$ enhances the expression of matrix metalloproteinase-2 [35]. Third, integrins $\alpha$ and $\beta$, which are induced by HIF, stimulate the proliferation and adhesion of endothelial cells, and HIF- $1 \alpha$ controls the growth of endothelial cells. The final step of angiogenesis is vascular maturation, which involves the recruitment of vascular sustentacular cells and the formation of the basement membrane.

The treatment goal of angiogenesis is achieved by stimulating the formation of new capillaries via the regulation of angiogenic growth factors. A variety of angiogenic growth factors, such as vascular growth factor (VEGF), placental growth factor, fibroblast growth factor, and platelet-derived growth factor, have been used in in vitro studies and preclinical and clinical trials [36-39]. Of these, VEGF may the most important growth factor involved in angiogenesis. The mammalian genome encodes five members of the VEGF family that regulate angiogenesis and lymphangiogenesis, namely, VEGF-A (also known as VEGF), PlGF, VEGF-B, VEGF-C, and VEGF-D [40-43]. In particular, VEGF-A plays a key role in the early stages of angiogenesis. Homozygous and heterozygous VEGF-A knockout mice have an embryonic lethal phenotype. Immaturity of the vascular system suggested that the local embryonic vascular concentration is appropriate, and the maturation of neovascularization can be promoted
[44, 45]. VEGF-A, VEGF-B, VEGF-C, and VEGF-D are the main factors involved in angiogenesis [46]. Budding and proliferation of new blood vessels are complex processes that involve a wide range of angiogenic factors and their receptors. Under hypoxic conditions, HIF-1 is the main factor upregulating VEGF expression [47, 48]. VEGF induces the expression of FMS-related tyrosine kinase and kinasebinding domain receptors, through paracrine mechanisms $[49,50]$. In addition, VEGF specifically and strongly upregulates expression of the plasminogen activator, degrades the basement membrane and matrix of small vessels, increases the permeability of the blood vessels for macromolecules, and promotes the extension, reproduction, migration (through the basement membrane), adherence, and alignment of endothelial cells under the function of chemotactic factors, resulting in the production of open tube-like structures [51-53]. These buds then fuse to form annular vascular branches, containing three-dimensional tubular structures. Perivascular cells constitute the main vascular structure and enable maturation of the collateral circulation, thereby forming a smooth pathway, allowing self-bridging, aiding blood circulation, and alleviating myocardial ischemia $[54,55]$. In conclusion, various in vitro and in vivo studies have demonstrated that VEGF promotes angiogenesis $[56,57]$.

miR-101 inhibits angiogenesis by targeting enhancer of zeste homolog 2 in tumor tissues [58, 59].Contrarily, Kim et al. found that miR-101 upregulates HO-1 and VEGF by targeting the Cullin 3 protein under hypoxic conditions, thereby promoting angiogenesis [10]. There are similar contradictory reports of the effects of miR-20a, a member of the miR-17-92 cluster, on angiogenesis. Inhibition of miR-20a reportedly promotes $[60,61]$ or inhibits [62] endothelial cell proliferation and migration. The aim of the current study was to investigate the ability of miR-101 to promote angiogenesis under hypoxic conditions. We found that transfection of HUVECs with agomiR-101 or EIF4E3 siRNA effectively 

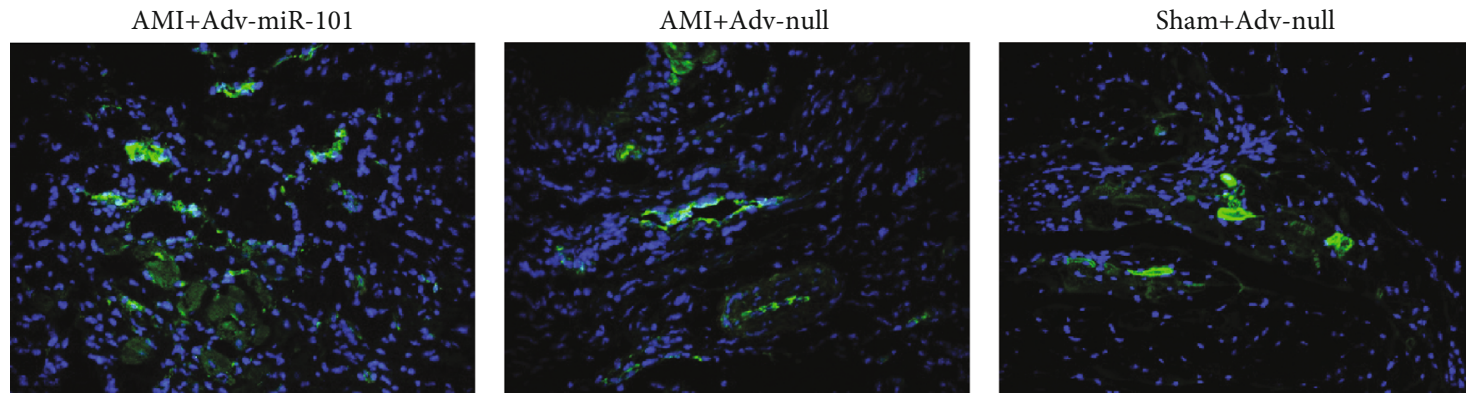

CD31

(a)

$\mathrm{AMI}+\mathrm{Adv}-\mathrm{miR}-101$

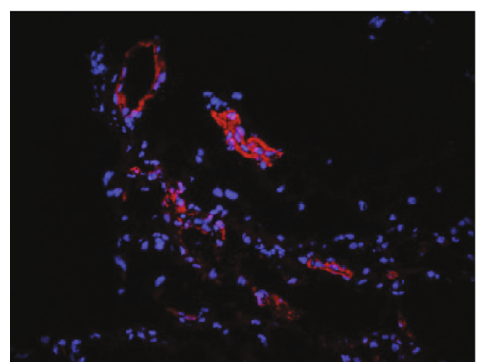

$\alpha$-SMA

DAPI
AMI+Adv-null

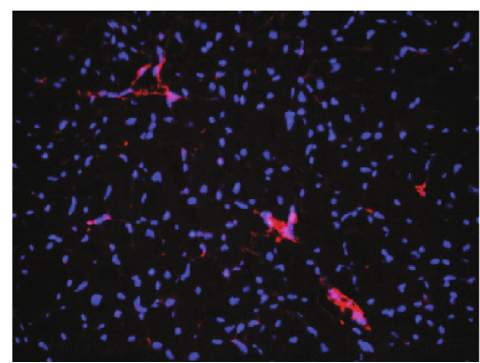

Sham+Adv-null

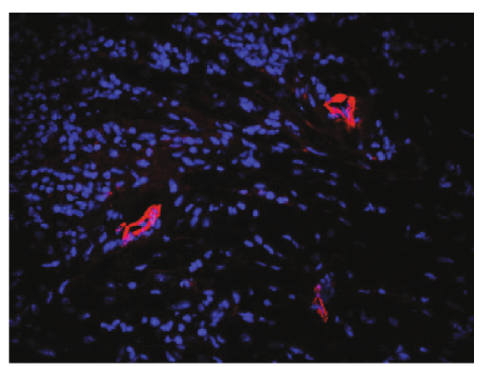

(b)

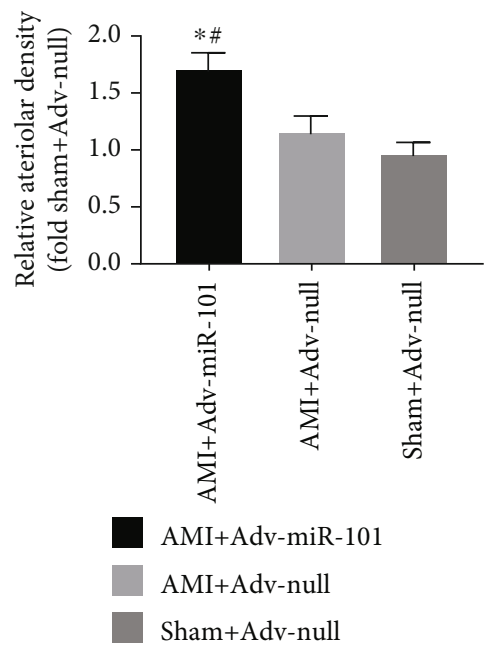

(c)

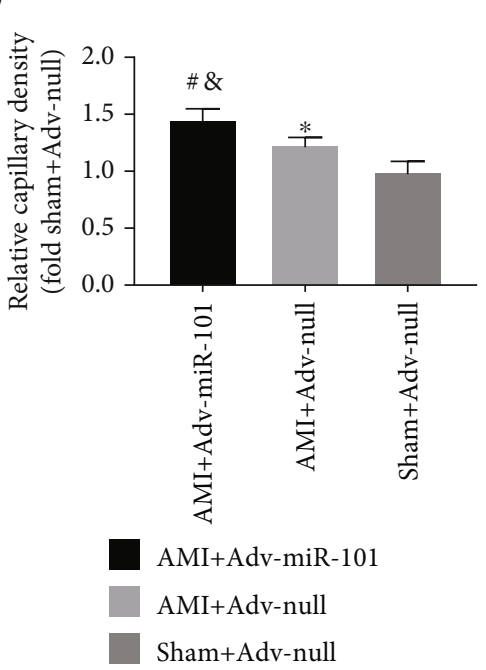

(d)

Figure 8: Photomicrographs (a, b) and bar graphs (c, d) show the vessel density and arteriole density of the peripheral infarcted myocardium $\left({ }^{*} P<0.05\right.$ vs. Sham + Adv-null; ${ }^{\&} P<0.01$ vs. Sham + Adv-null; ${ }^{\#} P<0.05$ vs. AMI + Adv-null).

inhibited expression of EIF4E3, and upregulated the HIF-1 $\alpha$ signaling pathway. VEGF-A expression was higher in cells expressing a combination of agomiR-101 and the EIF4E3specific siRNA than in those expressing the EIF4E3-specific siRNA alone, suggesting that miR-101 may also promote angiogenesis via other pathways, which is consistent with results from the study by Kim et al. [10].

The EIF4E family of eukaryotic translation initiation factors comprises EIF4E1, EIF4E2, and EIF4E3. Previous studies demonstrated that EIF4E1 plays an important role in the translation of HIF- $1 \alpha[24,25]$, promotes transcription of the HIF-1 $\alpha$ gene, activates the HIF-1 signaling pathway, stimulates the secretion of VEGF-A, and promotes angiogenesis [63]. EIF4E3 competitively inhibits the expression of EIF4E1 $[15,16]$. Moreover, EIF4E1 promotes the transcription of HIF- $1 \alpha$ mRNA into HIF- $1 \alpha$ [25], which may play an important role in angiogenesis. Therefore, by inhibiting the expression of EIF4E3 and stimulating the HIF-1 $\alpha$ 
signaling pathway, thereby promoting the formation and development of new capillaries, miR-101 plays an important role in stimulating the repair and regeneration of the blood vessels. EIF4E3 was identified as a potential target of miR101 using various bioinformatics packages. Subsequently, by using a luciferase reporter fused to the $3^{\prime}$-UTR of the EIF4E3 gene, we verified that miR-101 could directly regulate EIF4E3. In a HUVEC model of AMI, overexpression of miR101 inhibited the expression of EIF4E3, thereby promoting HIF- $1 \alpha$ expression and stimulating an increase in VEGF-A levels. Furthermore, in EdU, Transwell, and angiogenesis experiments, overexpression of miR-101 effectively enhanced endothelial cell proliferation, migration, and angiogenesis. These findings support our hypothesis that inhibition of EIF4E3 by miR-101 promotes the expression of EIF4E1, thereby enhancing activation of the HIF-1 signaling pathway, stimulating VEGF-A secretion, and promoting the proliferation, differentiation, and migration of endothelial cells. This assumption was further verified using a C57BL/6 AMI mouse model, established by ligating the left anterior descending coronary artery. The mice were then infected with a recombinant adenovirus vector Adv-miR-101. In animal experiments, miR101 effectively promoted the proliferation of vascular endothelial cells, increased the vascular density after AMI, and improved the ejection fraction, as well as the left ventricular short-axis systolic rate, left ventricular end-systolic diameter, and recovery of cardiac pumping function. However, the recovered myocardial function in mice in the experimental group differed from that of the sham-operated mice. These results indicate that miR-101 can effectively promote angiogenesis and cardiac function recovery in myocardial infarction areas in C57BL/6 mice; however, further studies are needed to confirm that miR-101 can be used to achieve complete recovery of cardiac function following AMI. Kim et al. [10] reported that miR-101 plays an important role in the repair and regeneration of the blood vessels during ischemia using a C57BL/6 mouse hindlimb ischemia model. Kim's group found that miR-101 inhibits the expression of Cullin 3 and activates the expression of HO-1, VEGF, eNOS, and other angiogenesisrelated factors, thus reversing ischemia-reperfusion injury. Moreover, there are also contradictory reports of the effects of miR-20a, a member of the miR-17-92 cluster, on angiogenesis. Inhibition of miR-20a reportedly promotes $[60,61]$ or inhibits [62] endothelial cell proliferation and migration. To the best of our knowledge, the current study is the first report that miR-101 plays a role in AMI, although we recognize that additional experiments are required to further our findings.

To summarize, in the present study, miR-101 expression in HUVECs was increased under hypoxic conditions, which is consistent with the results from Kim et al. [10]. These results may provide new insight for studying the pathogenesis of AMI and may provide novel targets for the diagnosis and treatment of this condition.

\section{Data Availability}

The data used to support the findings of this study are included within the article.

\section{Conflicts of Interest}

The authors declare no conflicts of interest.

\section{Authors' Contributions}

Jun Pang, Liwen Ye, and Qingwei Chen designed the experiments. Jun Pang, Liwen Ye, Jian Wang, Xixi Yang, and Lan Hao performed the experiments. Jun Pang, Liwen Ye, and Wuyang He analyzed the data. Jun Pang and Liwen Ye wrote the manuscript. All authors approved the final version of the manuscript. Jun Pang and Liwen Ye made equal contributions to this work.

\section{Acknowledgments}

The authors would like to extend their gratitude to Zhiqin $\mathrm{Wu}$ for his assistance with the angiogenesis assay. This study was undertaken at the Institute of Ultrasound Imaging of Chongqing Medical University and the State Key Laboratory of Ultrasound Engineering in Medicine Co-Founded by Chongqing and the Ministry of Science and Technology. The study work was supported by the National Natural Science Foundation of China (Grant Nos. 31871182 and 31600957). This study is funded by the Special Project of Science and Technology Strategic Cooperation between the municipal government and universities in Nanchong (Grant no. 18SXHZ0159).

\section{References}

[1] H. D. White and D. P. Chew, "Acute myocardial infarction," The Lancet, vol. 372, no. 9638, pp. 570-584, 2008.

[2] K. Regula and L. Kirshenbaum, "Apoptosis of ventricular myocytes: a means to an end," Journal of Molecular and Cellular Cardiology, vol. 38, no. 1, pp. 3-13, 2005.

[3] C. Cochain, K. M. Channon, and J. S. Silvestre, "Angiogenesis in the infarcted myocardium," Antioxidants \& Redox Signaling, vol. 18, no. 9, pp. 1100-1113, 2013.

[4] A. Longchamp, T. Mirabella, A. Arduini et al., "Amino acid restriction triggers angiogenesis via GCN2/ATF4 regulation of VEGF and H2S production," Cell, vol. 173, no. 1, pp. 117129.e14, 2018.

[5] L. Rao, K. De Veirman, D. Giannico et al., "Targeting angiogenesis in multiple myeloma by the VEGF and HGF blocking DARPin ${ }^{\circledR}$ protein MP0250: a preclinical study," Oncotarget, vol. 9, no. 17, pp. 13366-13381, 2018.

[6] C. W. Chen, L. L. Wang, S. Zaman et al., "Sustained release of endothelial progenitor cell-derived extracellular vesicles from shear-thinning hydrogels improves angiogenesis and promotes function after myocardial infarction," Cardiovascular Research, vol. 114, no. 7, pp. 1029-1040, 2018.

[7] A. L. Laiva, R. M. Raftery, M. B. Keogh, and F. J. O'Brien, "Proangiogenic impact of SDF- $1 \alpha$ gene-activated collagen-based scaffolds in stem cell driven angiogenesis," International Journal of Pharmaceutics, vol. 544, no. 2, pp. 372-379, 2018.

[8] A. V. Orang and A. Barzegari, "MicroRNAs in colorectal cancer: from diagnosis to targeted therapy," Asian Pacific Journal of Cancer Prevention, vol. 15, no. 17, pp. 6989-6999, 2014.

[9] A. K. Y. Tsui, P. A. Marsden, C. D. Mazer et al., "Differential HIF and NOS responses to acute anemia: defining organ- 
specific hemoglobin thresholds for tissue hypoxia," American Journal of Physiology-Regulatory, Integrative and Comparative Physiology, vol. 307, no. 1, pp. R13-R25, 2014.

[10] J. H. Kim, K. S. Lee, D. K. Lee et al., "Hypoxia-responsive microRNA-101 promotes angiogenesis via heme oxygenase$1 /$ vascular endothelial growth factor axis by targeting cullin 3," Antioxidants \& Redox Signaling, vol. 21, no. 18, pp. 2469-2482, 2014.

[11] S. Bian, L. Zhang, L. Duan, X. Wang, Y. Min, and H. Yu, "Extracellular vesicles derived from human bone marrow mesenchymal stem cells promote angiogenesis in a rat myocardial infarction model," Journal of Molecular Medicine, vol. 92, no. 4, pp. 387-397, 2014.

[12] B. H. Zhang, C. X. Guo, H. X. Wang et al., "Cardioprotective effects of adipokine apelin on myocardial infarction," Heart and Vessels, vol. 29, no. 5, pp. 679-689, 2014.

[13] J. Fiedler, V. Jazbutyte, B. C. Kirchmaier et al., "MicroRNA-24 regulates vascularity after myocardial infarction," Circulation, vol. 124, no. 6, pp. 720-730, 2011.

[14] J. I. Bardos and M. Ashcroft, "Negative and positive regulation of HIF-1: a complex network," Biochimica et Biophysica Acta (BBA) - Reviews on Cancer, vol. 1755, no. 2, pp. 107-120, 2005.

[15] L. Volpon, M. J. Osborne, B. Culjkovic-Kraljacic, and K. L. B. Borden, "eIF4E3, a new actor in mRNA metabolism and tumor suppression,” Cell Cycle, vol. 12, no. 8, pp. 1159-1160, 2014.

[16] M. J. Osborne, L. Volpon, J. A. Kornblatt, B. Culjkovic-Kraljacic, A. Baguet, and K. L. B. Borden, "eIF4E3 acts as a tumor suppressor by utilizing an atypical mode of methyl-7guanosine cap recognition," Proceedings of the National Academy of Sciences of the United States of America, vol. 110, no. 10, pp. 3877-3882, 2013.

[17] J. D. Cha, H. J. Kim, and I. H. Cha, "Genetic alterations in oral squamous cell carcinoma progression detected by combining array-based comparative genomic hybridization and multiplex ligation-dependent probe amplification," Oral Surgery, Oral Medicine, Oral Pathology, Oral Radiology, and Endodontics, vol. 111, no. 5, pp. 594-607, 2011.

[18] A. De Benedetti and J. R. Graff, "eIF-4E expression and its role in malignancies and metastases," Oncogene, vol. 23, no. 18, pp. 3189-3199, 2004.

[19] J. R. Graff and S. G. Zimmer, "Translational control and metastatic progression: enhanced activity of the mRNA capbinding protein eIF-4E selectively enhances translation of metastasis-related mRNAs," Clinical \& Experimental Metastasis, vol. 20, no. 3, pp. 265-273, 2003.

[20] R. B. Kjellerup, L. Iversen, K. Kragballe, and C. Johansen, “The expression, and phosphorylation of eukaryotic initiation factor 4E are increased in lesional psoriatic skin," The British Journal of Dermatology, vol. 161, no. 5, pp. 1059-1066, 2009.

[21] G. Wang, Z. Li, Z. Li et al., "Targeting eIF4E inhibits growth, survival and angiogenesis in retinoblastoma and enhances efficacy of chemotherapy," Biomedicine \& Pharmacotherapy, vol. 96, pp. 750-756, 2017.

[22] A. L. Landon, P. A. Muniandy, A. C. Shetty et al., "MNKs act as a regulatory switch for eIF4E1 and eIF4E3 driven mRNA translation in DLBCL," Nature Communications, vol. 5, no. 1, p. 5413, 2014.

[23] S. J. Cho, I. F. Teng, M. Zhang et al., "Hypoxia-inducible factor 1 alpha is regulated by RBM38, a RNA-binding protein and a p53 family target, via mRNA translation," Oncotarget, vol. 6, no. 1, pp. 305-316, 2015.
[24] J. Cao, L. He, G. Lin et al., "Cap-dependent translation initiation factor, eIF4E, is the target for Ouabain-mediated inhibition of HIF-1 $\alpha$," Biochemical Pharmacology, vol. 89, no. 1, pp. 20-30, 2014.

[25] T. Yi, E. Papadopoulos, P. R. Hagner, and G. Wagner, "Hypoxia-inducible factor- $1 \alpha$ (HIF-1 $\alpha$ ) promotes cap-dependent translation of selective mRNAs through up-regulating initiation factor eIF4E1 in breast cancer cells under hypoxia conditions," The Journal of Biological Chemistry, vol. 288, no. 26, pp. 18732-18742, 2013.

[26] Y. Y. Kim, L. Von Weymarn, O. Larsson et al., "Eukaryotic initiation factor $4 \mathrm{E}$ binding protein family of proteins: sentinels at a translational control checkpoint in lung tumor defense," Cancer Research, vol. 69, no. 21, pp. 8455-8462, 2009.

[27] G. L. Semenza and G. L. Wang, "A nuclear factor induced by hypoxia via de novo protein synthesis binds to the human erythropoietin gene enhancer at a site required for transcriptional activation," Molecular and Cellular Biology, vol. 12, no. 12, pp. 5447-5454, 1992.

[28] G. L. Wang and G. L. Semenza, "Characterization of hypoxiainducible factor 1 and regulation of DNA binding activity by hypoxia," The Journal of Biological Chemistry, vol. 268, no. 29, pp. 21513-21518, 1993.

[29] R. J. Kewley, M. L. Whitelaw, and A. Chapman-Smith, "The mammalian basic helix-loop-helix/PAS family of transcriptional regulators," The International Journal of Biochemistry \& Cell Biology, vol. 36, no. 2, pp. 189-204, 2004.

[30] G. L. Wang, B. H. Jiang, E. A. Rue, and G. L. Semenza, "Hypoxia-inducible factor 1 is a basic-helix-loop-helix-PAS heterodimer regulated by cellular $\mathrm{O} 2$ tension," Proceedings of the National Academy of Sciences of the United States of America, vol. 92, no. 12, pp. 5510-5514, 1995.

[31] Y. Makino, R. Cao, K. Svensson et al., "Inhibitory PAS domain protein is a negative regulator of hypoxia-inducible gene expression," Nature, vol. 414, no. 6863, pp. 550-554, 2001.

[32] M. Ema, S. Taya, N. Yokotani, K. Sogawa, Y. Matsuda, and Y. Fujii-Kuriyama, "A novel bHLH-PAS factor with close sequence similarity to hypoxia-inducible factor lalpha regulates the VEGF expression and is potentially involved in lung and vascular development," Proceedings of the National Academy of Sciences of the United States of America, vol. 94, no. 9, pp. 4273-4278, 1997.

[33] Y. Z. Gu, S. M. Moran, J. B. Hogenesch, L. Wartman, and C. A. Bradfield, "Molecular characterization and chromosomal localization of a third alpha-class hypoxia inducible factor subunit, HIF3alpha," Gene Expression, vol. 7, no. 3, pp. 205-213, 1998.

[34] P. Carmeliet and R. K. Jain, "Molecular mechanisms and clinical applications of angiogenesis," Nature, vol. 473, no. 7347, pp. 298-307, 2011.

[35] Y. Ben-Yosef, A. Miller, S. Shapiro, and N. Lahat, "Hypoxia of endothelial cells leads to MMP-2-dependent survival and death," American Journal of Physiology-Cell Physiology, vol. 289, no. 5, pp. C1321-C1331, 2005.

[36] A. Zimna, A. Janeczek, N. Rozwadowska et al., "Biological properties of human skeletal myoblasts genetically modified to simultaneously overexpress the pro-angiogenic factors vascular endothelial growth factor-A and fibroblast growth factor-4," Journal of Physiology and Pharmacology, vol. 65, no. 2, pp. 193-207, 2014.

[37] S. Kolakowski, M. F. Berry, P. Atluri et al., "Placental growth factor provides a novel local angiogenic therapy for ischemic 
cardiomyopathy," Journal of Cardiac Surgery, vol. 21, no. 6, pp. 559-564, 2006.

[38] S. Rajagopalan, E. R. Mohler III, R. J. Lederman et al., "Regional angiogenesis with vascular endothelial growth factor in peripheral arterial disease: a phase II randomized, double-blind, controlled study of adenoviral delivery of vascular endothelial growth factor 121 in patients with disabling intermittent claudication," Circulation, vol. 108, no. 16, pp. 1933-1938, 2003.

[39] R. J. Lederman, F. O. Mendelsohn, R. D. Anderson et al., "Therapeutic angiogenesis with recombinant fibroblast growth factor-2 for intermittent claudication (the TRAFFIC study): a randomised trial," The Lancet, vol. 359, no. 9323, pp. 20532058, 2002.

[40] M. Shibuya, "Involvement of Flt-1 (VEGF receptor-1) in cancer and preeclampsia," Proceedings of the Japan Academy. Series B, Physical and Biological Sciences, vol. 87, no. 4, pp. 167-178, 2011.

[41] N. Ferrara and R. S. Kerbel, "Angiogenesis as a therapeutic target,” Nature, vol. 438, no. 7070, pp. 967-974, 2005.

[42] M. Shibuya and L. Claesson-Welsh, "Signal transduction by VEGF receptors in regulation of angiogenesis and lymphangiogenesis," Experimental Cell Research, vol. 312, no. 5, pp. 549-560, 2006.

[43] K. Alitalo and P. Carmeliet, "Molecular mechanisms of lymphangiogenesis in health and disease," Cancer Cell, vol. 1, no. 3, pp. 219-227, 2002.

[44] N. Ferrara, K. Carver-Moore, H. Chen et al., "Heterozygous embryonic lethality induced by targeted inactivation of the VEGF gene," Nature, vol. 380, no. 6573, pp. 439-442, 1996.

[45] P. Carmeliet, V. Ferreira, G. Breier et al., "Abnormal blood vessel development and lethality in embryos lacking a single VEGF allele," Nature, vol. 380, no. 6573, pp. 435-439, 1996.

[46] J. E. Park, G. A. Keller, and N. Ferrara, "The vascular endothelial growth factor (VEGF) isoforms: differential deposition into the subepithelial extracellular matrix and bioactivity of extracellular matrix-bound VEGF," Molecular Biology of the Cell, vol. 4, no. 12, pp. 1317-1326, 1993.

[47] Y. Liu, S. R. Cox, T. Morita, and S. Kourembanas, "Hypoxia regulates vascular endothelial growth factor gene expression in endothelial cells. Identification of a 5 ' enhancer," Circulation Research, vol. 77, no. 3, pp. 638-643, 1995.

[48] J. A. Forsythe, B. H. Jiang, N. V. Iyer et al., "Activation of vascular endothelial growth factor gene transcription by hypoxiainducible factor 1," Molecular and Cellular Biology, vol. 16, no. 9, pp. 4604-4613, 1996.

[49] H. P. Gerber, F. Condorelli, J. Park, and N. Ferrara, “Differential transcriptional regulation of the two vascular endothelial growth factor receptor genes. Flt-1, but not Flk-1/KDR, is up-regulated by hypoxia," The Journal of Biological Chemistry, vol. 272, no. 38, pp. 23659-23667, 1997.

[50] J. Waltenberger, U. Mayr, S. Pentz, and V. Hombach, "Functional upregulation of the vascular endothelial growth factor receptor KDR by hypoxia," Circulation, vol. 94, no. 7, pp. 1647-1654, 1996.

[51] Y. Wang, Y. D. Zhu, Q. Gui, X. D. Wang, and Y. X. Zhu, "Glucagon-induced angiogenesis and tumor growth through the HIF-1-VEGF-dependent pathway in hyperglycemic nude mice," Genetics and Molecular Research, vol. 13, no. 3, pp. 7173-7183, 2014.
[52] M. Weber, F. Sennlaub, E. Souied et al., "Review and expert opinion in age related macular degeneration. Focus on the pathophysiology, angiogenesis and pharmacological and clinical data," Journal Français d'Ophtalmologie, vol. 37, no. 7, pp. 566-579, 2014.

[53] H. Chen, D. Liu, Z. Yang et al., "Adrenergic signaling promotes angiogenesis through endothelial cell-tumor cell crosstalk," Endocrine-Related Cancer, vol. 21, no. 5, pp. 783-795, 2014.

[54] K. Yamasaki, T. Nakasa, S. Miyaki, T. Yamasaki, Y. Yasunaga, and M. Ochi, "Angiogenic microRNA-210 is present in cells surrounding osteonecrosis," Journal of Orthopaedic Research, vol. 30, no. 8, pp. 1263-1270, 2012.

[55] Y. L. Lou, F. Guo, F. Liu et al., "miR-210 activates notch signaling pathway in angiogenesis induced by cerebral ischemia," Molecular and Cellular Biochemistry, vol. 370, no. 1-2, pp. 45-51, 2012.

[56] T. K. Lee, H. Hwang, K. S. Na et al., "Effect of angiogenesis induced by consecutive intramuscular injections of vascular endothelial growth factor in a hindlimb ischemic mouse model," Nuclear Medicine and Molecular Imaging, vol. 48, no. 3, pp. 225-229, 2014.

[57] S. Moens, J. Goveia, P. C. Stapor, A. R. Cantelmo, and P. Carmeliet, "The multifaceted activity of VEGF in angiogenesis - implications for therapy responses," Cytokine \& Growth Factor Reviews, vol. 25, no. 4, pp. 473-482, 2014.

[58] M. Smits, J. Nilsson, S. E. Mir et al., "miR-101 is downregulated in glioblastoma resulting in $\mathrm{EZH} 2$-induced proliferation, migration, and angiogenesis," Oncotarget, vol. 1, no. 8, pp. 710-720, 2010.

[59] M. Smits, S. E. Mir, R. J. A. Nilsson et al., "Down-regulation of miR-101 in endothelial cells promotes blood vessel formation through reduced repression of EZH2," PLoS One, vol. 6, no. 1, article e16282, 2011.

[60] C. Doebele, A. Bonauer, A. Fischer et al., "Members of the microRNA-17-92 cluster exhibit a cell-intrinsic antiangiogenic function in endothelial cells," Blood, vol. 115, no. 23, pp. 49444950, 2010.

[61] A. L. Pin, F. Houle, M. Guillonneau, E. R. Paquet, M. J. Simard, and J. Huot, "miR-20a represses endothelial cell migration by targeting MKK3 and inhibiting p38 MAP kinase activation in response to VEGF," Angiogenesis, vol. 15, no. 4, pp. 593608, 2012.

[62] Y. Suarez, C. Fernandez-Hernando, J. Yu et al., "Dicer-dependent endothelial microRNAs are necessary for postnatal angiogenesis," Proceedings of the National Academy of Sciences of the United States of America, vol. 105, no. 37, pp. 1408214087, 2008.

[63] Y. Chen, X. Jin, Z. Zeng, W. Liu, B. Wang, and H. Wang, "Estrogen-replacement therapy promotes angiogenesis after acute myocardial infarction by enhancing SDF-1 and estrogen receptor expression," Microvascular Research, vol. 77, no. 2, pp. 71-77, 2009. 